\title{
A critical analysis of the reported association between vasectomy and frontotemporal dementia
}

\author{
Tobias S Köhler ${ }^{1}$, Jeremy T Choy ${ }^{2}$, Anees A Fazili ${ }^{3}$, Joel F Koenig ${ }^{1}$ and Robert E Brannigan ${ }^{2}$ \\ Asian Journal of Andrology (2012) 14, 903-904; doi:10.1038/aja.2012.94; published online 15 October 2012
}

Dear Editor,

Researchers reported an association between vasectomy and primary progressive aphasia (PPA—a rare variety of frontotemporal dementia) in a 2006 manuscript in Cognitive Behavioral Neurology detailing a case-control study that compared 47 men with the disease to 57 controls. ${ }^{1}$ The rate of vasectomy in PPA patients was $40 \%$ (19/ 47) vs. $16 \%(9 / 57)$ in normal controls. This difference met statistical significance $(P=0.02)$. The study also found that there was a younger age of PPA onset for patients who had undergone vasectomy (58.8 vs. 62.9 years; $P=0.03$ ).

Inspired by one patient's perception of his PPA disease process, these researchers tested a hypothesis of an association between vasectomy and PPA. However, despite proper methodology of case-control design, sound statistical analyses and self-acknowledgement of several of the study's weaknesses, whether the relationship that they reported on is causal or not remains unclear. A causal link between vasectomy and PPA would have important ramifications for public health and informed consent of vasectomy patients. Our own patients continue to raise concern over this reported association during vasectomy consultation. We thus seek to carefully evaluate the publication reporting this association between vasectomy and PPA, as well as the larger body of medical literature, to determine if the reported association has been further characterized or if causality has been proven.

\section{THE ROLE OF SELECTION BIAS}

Selection bias provides one possible explanation for the results of the Cognitive Behavioral Neurology manuscript and would limit its applicability to general clinical practice. Selection bias must be considered for the PPA case group in light of the 12 individuals identified with PPA out of the initial 59 (20\%) who were not included because their vasectomy status could not be verified. The possibility that these subjects differ from those included seems significant. It might have been possible that vasectomy history for these patients was not available due to lack of a partner or family to verify it. Subjects without a partner or family would seem less likely to use vasectomy as a means of family planning.

Differential utilization of health care may also play a role in selection bias in this study. PPA is a rare disorder and may often be misdiagnosed as another form of dementia, such as Alzheimer's disease, in those men of lower socioeconomic status; these men may thus be less likely to receive specialized medical care. Men of higher socioeconomic status may be more likely to receive a correct diagnosis of PPA in a specialized center. This population is also more likely to undergo a vasectomy. Furthermore, men with vasectomies generally have several children, and therefore may be more likely to be brought in by family for early signs or symptoms of dementia, thus facilitating the correct diagnosis of a rare disorder such as PPA.

\section{THE ROLE OF MISCLASSIFICATION BIAS}

Misclassification bias is a common challenge for retrospective epidemiological studies. In this study, the PPA subjects are more likely to have been placed in the incorrect classification of occurrence of vasectomy than are the control subjects. It would seem likely that the control group of mentally intact individuals, who are generally well educated and motivated to volunteer for a study would more accurately recount vasectomy occurrence in comparison to a patient with dementia. The PPA group had a similar educational level, but education level of the corroborating partners was not controlled. Due to the small sample size of the study, misclassification of even a small number of subjects could significantly impact the apparent association of PPA with vasectomy. Although in this study the possibility of misclassification was reduced by partner verification, it may still play a role due to the mental impairment of the subjects and retrospective nature of determining vasectomy status performed several decades earlier.

\section{THE ROLE OF CONFOUNDING}

One must also consider the potential effect of confounding on the Cognitive Behavioral Neurology manuscript. Although the authors do an excellent job matching their cases and controls according to age, race and level of education, the causal pathway of PPA ultimately remains unknown. Thus, confounding by an unknown variable may exist since the true risk factor for PPA cannot assuredly have been equally distributed between cases and controls. ${ }^{2}$

\section{THE ROLE OF BRADFORD HILL PRINCIPLES: CONSISTENCY AND BIOLOGICAL PLAUSIBILITY}

Of Bradford Hill's nine viewpoints to address 'before we cry causation', often emphasized are consistency across multiple studies and

${ }^{1}$ Division of Urology, Southern Illinois University School of Medicine, Springfield, IL 62794, USA; ${ }^{2}$ Department of Urology, Northwestern University, Feinberg School of Medicine, Chicago, IL 60611, USA and ${ }^{3}$ Department of Urology, University of Rochester Medical Center, Rochester, NY 14642, USA

Correspondence: RE Brannigan (r-brannigan@northwestern.edu)

Received: 14 June 2012; Revised: 5 July 2012; Accepted: 28 July 2012; Published online: 15 October 2012 
biological plausibility. ${ }^{3}$ Clearly, additional studies need to be performed to establish consistency of findings in the reported association between vasectomy and PPA. With regards to biological plausibility, the authors of the Cognitive Behavioral Neurology manuscript postulate that antisperm antibodies may be responsible for the development of PPA, especially in light of the fact that sperm is the only tissue outside of the central nervous system that expresses tau protein. ${ }^{4}$ The link between tauopathies and PPA remains complicated; however, to date, the majority of autopsied PPA brains have failed to reveal any evidence of tauopathy via immuno-histochemical techniques. ${ }^{5}$ Additionally, the presence of antisperm antibodies in vasectomized men has thus far not been definitively associated with any pathological processes aside from the desired risk of male infertility. ${ }^{6}$

\section{THE BURDEN OF PROOF}

The impact of reduction in vasectomy based on possible disease risk would be particularly damaging in developing countries where alternative birth control methods are less feasible and infant and maternal mortality rates are high. To further emphasize this point, far stronger data showing a correlation between prostate cancer (exceptionally more common than PPA) and vasectomy was evaluated by the World Health Organization in 1993, and this organization concluded that 'no known biological mechanism existed to explain any possible association between vasectomy and prostate cancer and that any causal relationship between the two was unlikely'. Therefore, no changes in family planning policies concerning vasectomy were justified. ${ }^{7}$ An alternative approach is to determine a number needed to treat/harm statistic, but this requires a disease prevalence rate. Unfortunately, no specific prevalence data on PPA are available at this time. However, the disease process' rarity can be conceptualized by the fact that Cognitive Neurology and Alzheimer's Disease Center only enrolled a total of 120 patients from 1996 through mid-2006, in spite of the center's particular focus and dedication to this disease process. By default, any number needed to harm statistic based on an extrapolated PPA odds ratio from the Cognitive Behavioral Neurology manuscript would be exceedingly high.

Finally, the characteristics of the PPA cohort seem unlikely if a strong causal relationship exists between vasectomy and PPA. Vasectomy is a relatively common procedure, yet PPA remains a very rare disease. Clearly many men undergo vasectomy without developing PPA. Perhaps most importantly, the original PPA cohort was divided almost evenly between men and women (59 men/61 women). It is true that the proposed immunological mechanism in the Cognitive Behavioral Neurology manuscript, antisperm antibodies, occurs in women and non-vasectomized men. However, the actual rate of spontaneous formation of serum antisperm antibodies in men without a known history of vasectomy or testicular trauma is exceedingly lowcited as only $1 \%$ of men with proven fertility, and 5\%-6\% of men complaining of infertility. This compares to a serum antisperm antibody rate of $60 \%-70 \%$ in men with a history of vasectomy. ${ }^{4,8}$ Men with vasectomy would represent the vast majority of cases in males since so few of the non-vasectomized men would have these antibodies. However, the Cognitive Behavioral Neurology manuscript reports $60 \%$ of its male PPA cases as having no history of vasectomy. The large proportion of non-vasectomized males with PPA weakens the argument for causality of vasectomy and antisperm antibodies for PPA.

In conclusion, the Cognitive Behavioral Neurology manuscript raises concerns regarding the possible association between vasectomy and dementia. However, it ultimately contains numerous limitations including the possible relevance of selection bias, data misclassification, confounding and lack of adherence to the Bradford Hill principles of consistency and biological plausibility. Given the lack of evidence demonstrating that vasectomy causes PPA, we conclude that vasectomy remains a safe method of contraception. To date, no studies have shown that vasectomy causes any significant, long-term, adverse health events. Considering the large public health benefit of vasectomy, the exceedingly rare prevalence of PPA and its poorly understood etiology, the burden of proof for causality should be clarified through further research before the medical community significantly alters its vasectomy practices.

\section{COMPETING FINANCIAL INTERESTS}

TSK is a recipient of research funding from Abbott, American Medical Systems, Hollister, and Medtronic, as well as a consultant for Actient, American Medical Systems, Coloplast, and Medtronic, and a speaker for Allergan and Auxilium. The remaining authors have no conflicts of interest to declare.

1 Weintraub S, Fahey C, Johnson N, Mesulam MM, Gitelman DR et al. Vasectomy in men with primary progressive aphasia. Cogn Behav Neurol 2006; 19: 190-3.

2 Howards SS, Peterson HB. Vasectomy and prostate cancer: chance, bias, or a causal relationship? JAMA 1993; 269: 913-4.

3 Phillips C, Goodman K. The missed lessons of Sir Austin Bradford Hill. Epidemiol Perspect Innov 2004; 1 : 3.

4 Linnet L. Clinical immunology of vasectomy and vasovasostomy. Urology 1983; 22: 101-14.

5 Mesulam MM. Primary progressive aphasia. Ann Neurol 2001; 49: 425-32.

6 Lipshultz LI, Howards SS, Niederberger CS. Infertility in the Male, Fourth Edition. New York: Cambridge University Press; 2009. p238.

7 Farley TM, Meirik O, Mehta S, Waites GM. The safety of vasectomy: recent concerns. Bull World Health Organ 1993; 71: 413-9.

8 Ansbacher R. Vasectomy: sperm antibodies. Fertil Steril 1973; 24: 788-92. 